\title{
Adipocyte-Specific Fatty Acid-Binding Protein (AFABP) and Chemerin in Association with Gestational Diabetes: A Case- Control Study
}

\author{
Maryam Mosavat $\left(\mathbb{D},{ }^{1}\right.$ Mitra Mirsanjari, ${ }^{2}$ Bashir A. Lwaleed $\mathbb{D},{ }^{3}$ Maherah Kamarudin $\mathbb{D}^{\mathbb{D}},{ }^{1}$ \\ and Siti Zawiah Omar $\mathbb{1}^{1}$ \\ ${ }^{1}$ Department of Obstetrics \& Gynecology, Faculty of Medicine, University of Malaya, Kuala Lumpur, Malaysia \\ ${ }^{2}$ Mazandaran University of Medical Sciences, Emam Khomeini Hospital, Fereidonkenar, Mazandaran, Iran \\ ${ }^{3}$ School of Health Sciences at the University of Southampton, UK \\ Correspondence should be addressed to Maryam Mosavat; maryam.mst57@um.edu.my
}

Received 18 February 2021; Accepted 21 April 2021; Published 28 April 2021

Academic Editor: Antonio Schiattarella

Copyright () 2021 Maryam Mosavat et al. This is an open access article distributed under the Creative Commons Attribution License, which permits unrestricted use, distribution, and reproduction in any medium, provided the original work is properly cited.

\begin{abstract}
Background. Adipocytokines participate in regulating the inflammatory response in glucose homeostasis and type 2 diabetes. However, among these peptides, the role of adipocyte-specific fatty-acid-binding protein (AFABP), chemerin, and secreted protein acidic and rich in cysteine (SPARC) in gestational diabetes (GDM) has not been fully investigated. Method. The maternal fasting level of adipocytokines of 53 subjects with GDM and 43 normal pregnant (NGDM) was measured using multiplex immunoassay at 24-28 weeks, before delivery, immediate postpartum, and 2-6 months postpuerperium. Results. Higher levels of AFABP were associated with a 3.7-fold higher risk of GDM. Low chemerin levels were associated with a 3.6fold higher risk of GDM. Interleukin-10 (IL-10) was inversely associated with the risk of GDM. SPARC had no association with GDM. AFABP was directly correlated to interleukin-6 $(r=0.50)$, insulin resistance index $(r=0.26)$, and body mass index $(r=0.28)$ and inversely correlated to C-reactive protein $(r=-0.27)$. Chemerin levels were directly and strongly correlated with IL-10 $(r=0.41)$ and interleukin-4 $(r=0.50)$ and inversely correlated to insulin resistance index $(r=-0.23)$ in GDM but not NGDM. In the longitudinal assessment, there were no significant differences in AFABP and chemerin concentrations of both studied groups. Conclusion. AFABP and chemerin were associated with a higher risk of GDM. These adipocytokines were related to insulin resistance, body mass index, and inflammation in pregnant women diagnosed with GDM.
\end{abstract}

\section{Background}

Adipose tissue plays a significant role in the pathophysiology and development of type 2 diabetes (T2DM) and gestational diabetes (GDM) [1-4]. GDM is a condition of abnormal maternal glucose tolerance that occurs for the first time in pregnancy and can be prevented by using insulin sensitizers [5]. GDM is associated with an increased risk for subsequent abnormal glucose tolerance later in life [6]. Adipose tissue is a metabolically dynamic tissue secreting adipocytokines (or adipokines) that possess endocrine and paracrine properties, which are involved in energy homeostasis, immune response and systemic inflammation, reproduction function, and blood pressure regulation [7]. Adipocytokines chemerin, AFABP (adipocyte-specific fatty acid-binding protein), interleukin-4 (IL-4), and interleukin-6 (IL-6) are enlisted in the regulation of insulin resistance and inflammation [8]. An increase in the level of several cytokines and in particular IL-6 has been reported in coronavirus disease-19 (COVID19) infection and increased the risk of adverse prenatal outcomes such as gestational diabetes $[9,10]$. The role of chemerin is a novel adipocytokine that plays a role in adipogenesis, energy metabolism, and inflammation [11]. Studies have shown that expression of both chemerin and its receptor was upregulated in fat tissue of animal model with obesity and T2DM, where its circulation was 
considerably related to characteristics of metabolic syndrome and obesity (e.g., circulating triglycerides, blood pressure, body fat content, and insulin resistance) in normoglycemic individuals [12]. Chemerin elevation has been shown to lead to insulin resistance in in vitro studies of human myocytes and to glucose reduction in in vivo studies of animals in obese mice [13]. Furthermore, vitamin D deficiency has been associated with higher levels of circulating inflammatory marker chemerin and low insulin sensitivity [14] and increase the risk of GDM $[15,16]$. The recent finding reported the protective effect of vitamin D3 supplementation on GDM via improving the antioxidant and inflammatory status and decreasing circulating chemerin level [17]. In serial measurements in early, mid, and late pregnancy, chemerin levels have been related to a noticeably increase in late pregnancy compared to early and midpregnancy stages [18]. However, there are conflicting results regarding chemerin circulation during GDM with its level being shown to be either elevated [19-22], unchanged [23-26], or reduced [27].

Secreted protein acidic and rich in cysteine (SPARC), a multifunctional matricellular peptide of $43 \mathrm{kDa}$, is related to the extracellular matrix and expressed largely in the basal lamina [28]. SPARC manages cell functions including cell adhesion, differentiation, and proliferation [29], where the protein has a broad range of biological effects [30]. SPARC is highly expressed in subcutaneous fat, and its production and excretion in adipose tissue are affected by fat mass, insulin, and glucose [31]. SPARC with profibrotic effects participate in metabolic dysregulation in obesity [31]. High SPARC expression in adipose tissue leads to insulin resistance [32]. Furthermore, higher levels of this peptide have been linked to T2DM, diabetic retinopathy, and nephropathy [32, 33]. In a recent cross-sectional study on normal pregnancy and GDM, SPARC levels were significantly correlated with inflammation and dyslipidemia [32]. This recent study showed that SPARC independently represented insulin resistance in late pregnancy, suggesting its possible role in the pathophysiology of GDM. AFABP belongs to the fatty acidbinding protein family which is highly expressed in adipose tissue [34]. AFABP is the largest cytosolic protein of mature adipocytes, accounting for roughly $6 \%$ of total cellular proteins [35]. This protein acts as a significant regulator of systemic insulin sensitivity and lipid and glucose metabolism [36-38], where AFABP concentrations are directly associated with indicators of metabolic syndrome and vascular disease [34]. High fasting AFABP serum levels have been shown to predict the risk of metabolic and vascular morbidity and mortality in T2DM $[34,39]$. It has also been reported that AFABP secretion promotes insulin resistance in male mice, where AFABP mediates the degradation of peroxisome proliferator-activated receptors $(\operatorname{PAAR} \gamma)$ in adipose tissue and consequently reduces the expression of insulinsensitizing adiponectin [40]. AFABP has been correlated with insulin resistance and inflammation in T2DM and associated obesity [41]. Upregulation of AFABP in GDM mothers has been described in previous cross-sectional studies which may be linked with obesity and insulin resistance in pregnancy $[42,43]$. A study on pregnant women has stated that fetal tissues are the main source of cord arterial serum
AFABP [42]. The same study also suggests that in fetuses of pregnancy with GDM, AFABP values correlate with adiposity indicators [42].

To the best of the authors' knowledge, no study has defined the predictive value of chemerin, SPARC, and AFABP concentrations in the development of GDM. Therefore, this study has been designed to assess the association between the serum concentration of these adipocytokines and the development of GDM and to evaluate the circulation of these peptides throughout pregnancy and after delivery.

\section{Method}

The protocol of this study has been approved by the Scientific Review Committee of University of Malaya Medical Centre (UMMC) (reference number 1052.8, MEC ID 2014020725), and written informed consent was obtained from all participants. The protocol of this study was published elsewhere [3, 4, 44]. Briefly, as the authors have described recently [3, 4, 44], pregnant women aged between 18 and 45 years, gestational age between 24 and 28 weeks, a singleton pregnancy, and intending to participate in our longitudinal study and delivery at UMMC were initially eligible to participate in this cohort study. Those women with multifetal pregnancy, history of pregestational diabetes or previous GDM, drug, smoke, and/or alcohol abuse, hypertension, heart disease, renal or liver disease, uncontrolled endocrine disease, or any medical conditions that would affect lipid and glucose metabolism were deemed not eligible to enter the study. At the end of the longitudinal study, pregnancy outcomes were abstracted by extracting information from medical records. Subjects from whom it was not possible to collect a fasting blood sample or those who developed any pregnancy complications such as preeclampsia, eclampsia, pregnancy-induced hypertension $(\mathrm{PIH})$, preterm labour ( $<36$ weeks), or postterm labour ( $>41$ weeks and 6 days) were also excluded from the study. The maternal fasting samples were collected on four occasions:

Examination 1 (E1): 24-28 weeks of pregnancy (at the time of GDM screening).

Examination 2 (E2): prior to caesarean/vaginal delivery.

Examination 3 (E3): early postpartum; within 24 hours after delivery.

Examination 4 (E4): postpuerperium; within 2-6 months after delivery.

Screening for the diagnosis of GDM was performed by the 2-hour $75 \mathrm{~g}$ oral glucose tolerance test; fasting plasma glucose level greater than or equal to $5.6 \mathrm{mmol} / \mathrm{L}$ or 2 -hour plasma glucose level greater than or equal to $7.8 \mathrm{mmol} / \mathrm{L}$ [45]. Serum levels of SPARC, chemerin, AFABP, IL-4, IL-6, IL-10, and CRP were measured using Magnetic Multiplex Sandwich ELISA assay (LXSAHM, R\&D, USA). Based on the manufacturer's report the intra-inter-assay coefficient of variation $(\mathrm{CV} \%)$ of the assays were as follows: adiponectin (5.6-9.2), chemerin (6.7-13.7), AFABP (4.7-13.8) and SPARC (5.0-12.0), IL-4 (6.3-10.27), IL-6 (5.2-9.6), IL-10 (5.4-10.73), and CRP [9-13]. Homeostasis model assessment index (HOMA-IR) was computed using the following formula: fasting serum glucose $(\mathrm{mmol} / \mathrm{L}) / 22.5 \times$ fasting serum insulin 
(mIU/L). Data regarding HOMA-IR has been reported previously [3].

2.1. Statistical Analysis. Descriptive statistics were applied for qualitative variables (mean \pm standard error $(\mathrm{SE})$ ). The parametric Student's $t$-test or nonparametric Mann-Whitney $U$ test was used to compare differences between two independent groups. Binary and multivariate logistic regression was performed to explore associations between the studied peptides and GDM risk (odds ratio (OR; 95\% confidence interval (CI)). A paired sample $t$-test or two-related samples Wilcoxon test was performed to assess longitudinal changes between different time points (using Bonferroni correction). The Spearman/Pearson correlation coefficient described the correlation between peptides and metabolic markers. The $p$ value of $<0.05$ was considered as statistically significant. All statistical analyses were performed using IBM SPSS 26.0.

\section{Results}

Ninety-six pregnant participants comprised of 53 who were diagnosed with GDM and 43 with normal pregnancy (NGDM) were recruited in this study. The demographic characteristics of the subjects are presented in Table 1. At the time of Examination 1, no significant differences were observed for participants' age $(p>0.24)$, pregestational BMI $(p=0.40)$, gestational BMI $(p>0.88)$, gestational weeks $(p=0.72)$, family history of diabetes $(p=0.07)$, and parity $(p=0.88)$ between both the GDM and NGDM groups. As expected, the GDM group presented a higher fasting blood glucose level $(p=0.003)$ and 2 hours postprandial glucose tolerance test $(p=0.006)$, as compared to NGDM subjects. Those diagnosed with GDM presented lower IL-10 $(p<0.001)$ and IL-4 $(p=0.04)$ compared to the normal group. No significant difference was observed in the level of IL-6 and CRP.

Using logistic regression, there was no association between SPARC, IL-4, IL-6, and CRP concentration and GDM risk (Table 2). IL-10 was inversely associated with the risk of GDM (0.18 (95\% CI: 0.07-0.45)). Serum AFABP was directly associated with GDM risk. Participants with the higher levels of AFABP $(>10.09 \mathrm{ng} / \mathrm{mL})$ had a 3.7-fold higher risk of developing GDM compared with the lowest level. However, this relationship was attenuated but remained significant after adjustment for confounders including maternal age, gestational weeks, and BMI (aOR 1.1, 95\% CI: 1.00-1.22). Serum chemerin (OR 0.85 (95\% CI: 0.73-0.98)) was inversely associated with GDM. In the crude analysis, participants with chemerin $(<8.03 \mathrm{ng} / \mathrm{mL})$ presented a 3.6-fold higher risk of GDM compared to participants with the $>10.2 \mathrm{ng} / \mathrm{mL}$ ( $95 \%$ CI: 1.3-10.4). After adjustment for maternal age, gestational age, and BMI, the lowest tertile of the chemerin value remained a strong predictor for the diagnosis of GDM (aOR 4.5; 95\% CI: 1.4-14.0) (Table 2).

Using Spearman/Pearson correlation coefficient, AFABP was directly correlated to IL-6 $(r=0.50)$, HOMA-IR $(r=0.26)$, BMI $(r=0.28)$, and CRP $(r=0.27)$. Serum chemerin level was directly and strongly correlated with IL-10
TABLE 1: Demographic characteristics of subjects, mean (SE).

\begin{tabular}{lcc}
\hline & GDM & NGDM \\
\hline Participants' age (year) & $33.2(0.6)$ & $32.1(0.8)$ \\
Gestational week & $25.8(0.2)$ & $25.9(0.2)$ \\
Pregestation BMI $\left(\mathrm{kg} / \mathrm{m}^{2}\right)$ & $27.0(0.8)$ & $25.2(0.7)$ \\
FBG $(\mathrm{mmol} / \mathrm{L})$ & $5.0(0.2)$ & $4.2(0.1)^{*}$ \\
$2 \mathrm{hrs} \mathrm{OGTT}(\mathrm{mmol} / \mathrm{L})$ & $10.8(1.5)$ & $5.9(0.1)^{*}$ \\
IL-10 $(\mathrm{pg} / \mathrm{mL})$ & $1.2(0.10)$ & $4.0(0.32)^{*}$ \\
IL-4 $(\mathrm{pg} / \mathrm{mL})$ & $6.5(0.31)$ & $8.8(1.67)^{*}$ \\
IL-6 $(\mathrm{pg} / \mathrm{mL})$ & $2.8(0.41)$ & $2.6(0.34)$ \\
CRP $(\mathrm{ng} / \mathrm{mL})$ & $2.8(0.86)$ & $2.8(0.33)$ \\
\hline
\end{tabular}

${ }^{*} p$ value $<0.05$ significant difference between the two pregnancy groups.

$(r=0.41)$ and IL-4 $(r=0.50)$ and inversely correlated to HOMA-IR $(r=-0.23)$ in GDM but not NGDM.

The intra- and intergroup comparisons of adipocytokines are shown in Table 3. Over the pregnancy and postpuerperium, no significant difference was observed in the SPARC level between both studied groups. Levels of AFABP concentration were high in GDM just in E1 $(p=0.04)$ compared to NGDM. AFABP was in the lowest level in the first examination; however, with advancement in gestational age, its levels increased $(p<0.0001)$ and reached a peak in late pregnancy in both GDM and NGDM. Immediately after delivery, the level of AFABP decreased $(p<0.002)$ and this reduction continued slightly in postpuerperium. Serum chemerin was significantly low in GDM compared to NGDM (E1: $p=0.02$ ). There was no significant difference between chemerin concentration of GDM and NGDM groups in late pregnancy and after delivery. In the longitudinal assessment, chemerin levels of the normal pregnant group decreased with pregnancy development $(p<0.05)$. This reduction continued slightly and reached to its lowest level in postpuerperium (E4). In contrast, there were no significant changes in chemerin concentration of GDM with progress in gestational age. However, its concentrations reduced slightly $(p>0.05)$ in E3 and reached to its lowest level $(p<0.05)$ in E4.

\section{Discussion}

In this study, we showed that higher circulation of fasting serum AFABP concentrations in the second trimester, at the time of GDM screening, was associated with an increased risk for the development of GDM. When we categorized AFABP concentration to the four quartiles, we noticed that participants of upper quartile with the AFABP level higher than $10.09 \mathrm{ng} / \mathrm{mL}$ were at risk of GDM about 3.7-fold higher than pregnant women in the lowest quartile (AFABP $<4.90 \mathrm{ng} / \mathrm{mL}$ ). This relationship is attenuated and reduced to about 1.1 but remained significant when we adjusted for confounders including maternal age, gestational week, and BMI. Similarly, a study on pregnant women in their first trimester of pregnancy reported an association of GDM risk with higher quartile AFABP concentration [42, $43,46]$. AFABP is highly expressed in adipocytes and contributes to insulin sensitivity and energy metabolism. We 
TABLe 2: Association between adipocytokines and GDM risk (24-28 weeks).

\begin{tabular}{|c|c|c|c|}
\hline & & Unadjusted OR (95\% CI) & Adjusted OR (95\% CI) \\
\hline IL-10 (pg/mL) & & $0.18(0.07-0.45)^{*}$ & $0.17(0.06-0.48)^{*}$ \\
\hline${ }^{\circ} p$ value & & $<0.001$ & 0.001 \\
\hline $\mathrm{IL}-4(\mathrm{pg} / \mathrm{mL})$ & & $0.98(0.96-1.01)$ & $0.98(0.95-1.00)$ \\
\hline IL-6 (pg/mL) & & $1.03(0.82-1.30)$ & $1.03(0.79-1.33)$ \\
\hline CRP (ng/mL) & & $1.0(1.0-1.01)$ & $1.0(1.0-1.01)$ \\
\hline SPARC $(\mu \mathrm{g} / \mathrm{mL})$ & & $0.44(0.16-1.20)$ & $0.12(0.16-1.24)$ \\
\hline \multicolumn{4}{|l|}{$\operatorname{AFABP}(\mathrm{ng} / \mathrm{mL})$} \\
\hline Quartile 1 & $X<4.90$ & Referent & Referent \\
\hline Quartile 2 & $4.90<X<7.57$ & $2.02(0.62-6.55)$ & $1.92(0.55-6.67)$ \\
\hline Quartile 3 & $7.57<X<10.09$ & $0.93(0.29-3.0)$ & $0.92(0.27-3.11)$ \\
\hline Quartile 4 & $X>10.09$ & $3.68(1.06-12.77)^{*}$ & $1.11(1.00-1.22)^{*}$ \\
\hline$p$ value & & 0.04 & 0.03 \\
\hline \multicolumn{4}{|c|}{ Chemerin $(\mathrm{ng} / \mathrm{mL})$} \\
\hline Tertile 1 & $<8.03$ & $3.6(1.3-10.4)$ & $4.5(1.4-14.0)$ \\
\hline Tertile 2 & $8.0 \leq X<10.2$ & $1.2(0.4-3.2)$ & $1.1(0.4-3.0)$ \\
\hline Tertile 3 & $\geq 10.2$ & Referent & Referent \\
\hline$p$ value & & $<0.001^{*}$ & $0.001^{*}$ \\
\hline
\end{tabular}

OR $(95 \% \mathrm{CI})$ adjusted for maternal age, gestational age, and BMI. ${ }^{*} p$ value $<0.05$.

TABLE 3: Between- and within-group comparisons of adipocytokine.

\begin{tabular}{|c|c|c|c|c|}
\hline & Examination (E1) & Examination (E2) & Examination (E3) & Examination (E4) \\
\hline \multicolumn{5}{|c|}{ SPARC $(\mu \mathrm{g} / \mathrm{mL})$} \\
\hline GDM & $1.23(0.07)$ & $1.09(0.06)^{\mathrm{a}}$ & $1.08(0.06)$ & $1.03(0.07)^{\mathrm{a}}$ \\
\hline NGDM & $1.37(0.05)$ & $1.11(0.05)^{\mathrm{a}}$ & $1.06(0.05)$ & $1.00(0.05)^{\mathrm{a}}$ \\
\hline$p$ value & 0.11 & 0.80 & 0.80 & 0.68 \\
\hline \multicolumn{5}{|c|}{$\operatorname{AFABP}(\mathrm{ng} / \mathrm{mL})$} \\
\hline GDM & $9.11 \pm 0.69$ & $13.57 \pm 1.13^{\mathrm{a}}$ & $12.47 \pm 1.19^{\mathrm{a}, \mathrm{b}}$ & $10.87(1.00)^{\mathrm{b}}$ \\
\hline NGDM & $7.25 \pm 0.52$ & $12.28 \pm 0.91^{\mathrm{a}}$ & $10.39 \pm 0.79^{\mathrm{a}, \mathrm{b}}$ & $8.94 \pm(0.68)^{\mathrm{a}, \mathrm{b}}$ \\
\hline$p$ value & 0.04 & 0.39 & 0.15 & 0.12 \\
\hline \multicolumn{5}{|c|}{ Chemerin $(\mathrm{ng} / \mathrm{mL})$} \\
\hline GDM & $8.70(0.46)$ & $8.17(0.38)$ & $7.76(0.32)$ & $6.18(0.35)^{\mathrm{a}, \mathrm{c}}$ \\
\hline NGDM & $10.12(0.35)$ & $8.14(0.34)^{\mathrm{a}}$ & $7.74(0.33)^{\mathrm{a}}$ & $5.79(0.25)^{\mathrm{a}, \mathrm{b}, \mathrm{c}}$ \\
\hline$p$ value & 0.02 & 0.94 & 0.97 & 0.40 \\
\hline
\end{tabular}

${ }^{\mathrm{a}} p$ value $<0.05$ compared to Examination $1 .{ }^{\mathrm{b}} p$ value $<0.05$ compared to Examination $2 .{ }^{\mathrm{c}} p$ value $<0.05$ compared to Examination $3 .{ }^{*} p$ value $<0.05$ compared to NGDM.

further observed that AFABP levels were positively associated with HOMA-IR and BMI. Similar to this finding, AFABP has recently been proposed as a marker of metabolic syndrome in nonpregnancy states [34, 47, 48]. In another study, higher AFABP concentration in GDM was also noted to be an independent risk factor for increased insulin resistance [49]. The role of AFABP in association with insulin resistance, inflammation, and obesity in T2DM has been noted previously $[41,50]$. Similarly, in this study, correlations between AFABP level and IL- 6 and CRP indicated a proinflammatory role of this adipokine in GDM.

In our longitudinal assessment, we observed that serum AFABP levels in both pregnant groups increased with gesta- tional age and then decreased immediately after delivery. This study is the first to evaluate longitudinal changes in serum AFABP in women with and without GDM during and after their pregnancy period. However, we only found one other study that reported a significant increase in AFABP from the second to the third trimester of pregnancy [43]. Based on a report by Ortega-Senovilla et al. [51], AFABP concentration of cord blood was shown to be higher than maternal level, suggesting that fetal tissues are the main source of AFAPB in cord blood. Hence, increased levels of AFABP in correlation with advanced gestational age and its reduction immediately after delivery implies a significant role of fetal tissues in AFABP production. 
In the present study, the second trimester of GDM pregnancy is linked to lower chemerin concentration than normal pregnant controls. Subsequently, we found that chemerin concentrations $\leq 8.0 \mathrm{ng} / \mathrm{mL}$ are associated with a 3.3 -fold increment in GDM risk compared to levels $\geq 10.2 \mathrm{ng} / \mathrm{mL}$. This result was relatively unchanged after adjusting for BMI and maternal and gestational age. Furthermore, we observed that the chemerin level of GDM was inversely and independently correlated to HOMA-IR. Pregnancy is a complex of metabolic changes in early pregnancy, followed by insulin resistance [52]. It has been determined that GDM develops in the presence of the inability of pancreatic $\beta$-cells to induce sufficient insulin secretion and counteract insulin insensitivity of tissues during pregnancy [21]. An animal study has shown that chemerin and its receptors are substantially expressed in $\beta$-cells which are necessary for insulin secretion in vitro and in vivo [53]. Subsequently, chemerin deficiency causes glucose intolerance mainly due to increased hepatic glucose production and impaired insulin secretion [53]. Hence, a reduction in chemerin levels is associated with the development of GDM through decreased insulin sensitivity and attenuated anti-inflammatory capacity [21]. Similar to our study, Hare et al. [27] proposed that low chemerin levels in GDM may lead to insulin resistance and a higher level in normal pregnancy, which may provide a protective effect to decrease pregnancy-induced insulin resistance. Serum levels of chemerin may also be influenced by multiple factors in relation to inflammation and/or metabolic states related to obesity. Lower chemerin level observed in GDM subjects in the current study was formed in correlation with a reduction in IL-10 level. IL-10, an anti-inflammatory cytokine and an immunosuppressive, has been discussed as a key regulator for inflammatory cytokines. Moreli et al. [54], in a study on pregnant women, showed that glycemic mean $\geq 100 \mathrm{mg} / \mathrm{dL}$ is associated with a reduction in maternal IL-10 concentrations. Vitamin D level has been associated with circulating inflammatory markers and chemerin and low insulin sensitivity [14] and increase the risk of GDM $[15,16]$. The protective effect of vitamin D3 supplementation on GDM through improving the antioxidant and inflammatory status and decreasing circulating chemerin level has been reported recently. In this study, as a limitation, we did not measure the level of vitamin. More studies are warranted to elucidate the relationship between vitamin $\mathrm{D}$ level and the inflammatory cytokines specially chemerin in GDM.

Interestingly, we also observed that pregnancy was associated with peak chemerin levels at 24-28 weeks of gestation. When pregnancy progressed, the differences between chemerin levels in both pregnancy groups diminished as serum chemerin levels in women with normal pregnancy decreased significantly, and the corresponding levels in GDM subjects remained relatively unchanged. We further noted an inverse independent correlation between chemerin levels and insulin resistance irrespective of maternal BMI levels. Our finding is in line with previous human and animal studies which revealed that chemerin levels are significantly reduced in circulating serum throughout pregnancy. This reduction has been shown to be inversely associated with a general increase in insulin resistance during gestation $[27,55]$. Therefore, unchanged levels of chemerin in GDM subjects in the present study may be due to appropriate managing of disease with diet and/or insulin therapy. This may also explain the comparable serum chemerin levels and normal glucose tolerance in late pregnancy observed in subjects with GDM. In addition, during pregnancy, adipocytokines are produced not only by adipose tissue but also by the placenta [56]. An animal study by Garces et al. [55] has shown that the expression of chemerin mRNA was at its highest level at day 16. This subsequently decreased significantly towards the end of the pregnancy, hence describing an anti-inflammatory environment. Their study also indicated that reductions in levels of chemerin in both the placenta and maternal circulation may be necessary for adequate maternal-fetal immune interaction, essential for the normal progress of pregnancy. Within 24 hours after delivery, chemerin levels insignificantly reduced and remained statistically unchanged in both studied groups. However, there was a significant reduction in chemerin levels postpuerperium in the presence of normal glucose tolerance reestablishment, which challenge the probable role of chemerin in the progression of T2DM after pregnancy.

In summary, the findings of this study suggest that AFABP and chemerin are associated with increased GDM risk, with $\mathrm{AFABP}$ and chemerin levels being related to insulin resistance, BMI, and inflammation in women diagnosed with GDM. However, in the nonpregnant state, these peptides had no further contribution to the development of metabolic syndrome when glucose tolerance was achieved. Additional studies with a large sample size are desired to confirm the findings of this study.

\section{Data Availability}

Data are available from the authors upon reasonable request.

\section{Conflicts of Interest}

There is no conflict of interest.

\section{Acknowledgments}

We greatly acknowledge the women who kindly participated in this study. This study was funded by the High Impact Research Grant of the Ministry of Higher Education, Universiti Malaya (UM.C/625/1/HIR/MOHE/MED/28).

\section{References}

[1] M. Wójcik, M. Chmielewska-Kassassir, K. Grzywnowicz, L. Woźniak, and K. Cypryk, "Związek hormonów pochodzących z tkanki tłuszczowej z cukrzycą ciążową (GDM)," Endokrynologia Polska, vol. 65, no. 2, pp. 134-142, 2014.

[2] M. Haluzík, D. Michalský, M. Kasalický et al., "Adipose tissue as a source of proinflammatory factors in obesity: the importance in the development of insulin resistance and diabetes," Collection of Czechoslovak Chemical Communications, vol. 9, pp. 49-52, 2015.

[3] M. Mosavat, S. Z. Omar, P. C. Tan, M. F. M. Razif, and P. Sthaneshwar, "Leptin and soluble leptin receptor in 
association with gestational diabetes: a prospective case-control study," Archives of Gynecology and Obstetrics, vol. 297, no. 3, pp. 797-803, 2018.

[4] M. Mosavat, S. Z. Omar, and P. Sthanshewar, "Serum FGF-21 and FGF-23 in association with gestational diabetes: a longitudinal case-control study," Hormone Molecular Biology and Clinical Investigation, vol. 41, 2020.

[5] G. Muscogiuri, S. Palomba, A. S. Laganà, and F. Orio, "Inositols in the treatment of insulin-mediated diseases," International Journal of Endocrinology, vol. 2016, Article ID 3058393, 2016.

[6] F. Corrado, R. D’Anna, A. S. Laganà, and A. Di Benedetto, "Abnormal glucose tolerance later in life in women affected by glucose intolerance during pregnancy," Journal of Obstetrics and Gynaecology, vol. 34, no. 2, pp. 123-126, 2014.

[7] P. Mancuso, "The role of adipokines in chronic inflammation," ImmunoTargets and Therapy, vol. 5, pp. 47-56, 2016.

[8] K. Makki, P. Froguel, and I. Wolowczuk, "Adipose tissue in obesity-related inflammation and insulin resistance: cells, cytokines, and chemokines," ISRN Inflammation, vol. 2013, Article ID 139239, 2013.

[9] N. Justman, G. Shahak, O. Gutzeit et al., "Lockdown with a price: the impact of the COVID-19 pandemic on prenatal care and perinatal outcomes in a tertiary care center," The Israel Medical Association Journal, vol. 22, no. 9, pp. 533-537, 2020.

[10] A. L. Fedullo, A. Schiattarella, M. Morlando et al., "Mediterranean diet for the prevention of gestational diabetes in the Covid-19 era: implications of Il-6 in diabesity," International Journal of Molecular Sciences, vol. 22, no. 3, p. 1213, 2021.

[11] A. A. Roman, S. D. Parlee, and C. J. Sinal, "Chemerin: a potential endocrine link between obesity and type 2 diabetes," Endocrine, vol. 42, no. 2, pp. 243-251, 2012.

[12] K. Bozaoglu, K. Bolton, J. McMillan et al., "Chemerin is a novel adipokine associated with obesity and metabolic syndrome," Endocrinology, vol. 148, no. 10, pp. 4687-4694, 2007.

[13] M. Fasshauer, M. Blüher, and M. Stumvoll, "Adipokines in gestational diabetes," The Lancet Diabetes and Endocrinology, vol. 2, no. 6, pp. 488-499, 2014.

[14] M. Reyman, A. A. Verrijn Stuart, M. van Summeren et al., "Vitamin D deficiency in childhood obesity is associated with high levels of circulating inflammatory mediators, and low insulin sensitivity," International Journal of Obesity, vol. 38, no. 1, pp. 46-52, 2014.

[15] M. Mosavat, D. Arabiat, A. Smyth, J. Newnham, and L. Whitehead, "Second-trimester maternal serum vitamin D and pregnancy outcome: the Western Australian Raine cohort study," Diabetes Research and Clinical Practice, vol. 175, no. article 108779, 2021.

[16] G. Rizzo, S. Garzon, M. Fichera et al., "Vitamin D and gestational diabetes mellitus: is there a link?," Antioxidants, vol. 8, no. 11, p. 511, 2019.

[17] A. Abeer and M. M. Suzan, "Vitamin D supplementation reduces serum chemerin level in gestational diabetes mellitus rat model," The Medical Journal of Cairo University, vol. 87, no. 9, pp. 3069-3080, 2019.

[18] M. F. Garces, E. Sanchez, A. I. Ruíz-Parra et al., "Serum chemerin levels during normal human pregnancy," Peptides, vol. 42, pp. 138-143, 2013.

[19] S. S. Fatima, F. Alam, B. Chaudhry, and T. A. Khan, "Elevated levels of chemerin, leptin, and interleukin-18 in gestational diabetes mellitus," The Journal of Maternal-Fetal \& Neonatal Medicine, vol. 30, no. 9, pp. 1023-1028, 2017.

[20] X.-M. Li, H. Ji, C.-J. Li, P.-H. Wang, P. Yu, and D.-M. Yu, "Chemerin expression in Chinese pregnant women with and without gestational diabetes mellitus," in Annales d'endocrinologie, pp. 19-24, Elsevier, 2015, http://www.sciencedirect.com/ science/article/pii/S0003426614010038.

[21] X. Yang, X. Quan, Y. Lan et al., "Serum chemerin level during the first trimester of pregnancy and the risk of gestational diabetes mellitus," Gynecological Endocrinology, vol. 33, pp. 1-4, 2017.

[22] A. Nazarian, F. Niknam, S. Mazloumzadeh, M. Kashanian, and S. Mazloumi, "The relation of serum chemerin levels with parameters of metabolic syndrome between pregnant women with gestational diabetes and normal pregnant women," Iranian Journal of Endocrinology and Metabolism (IJEM), vol. 14, no. 5 (SN 65), pp. 423-429, 2012.

[23] G. Barker, R. Lim, G. E. Rice, and M. Lappas, "Increased chemerin concentrations in fetuses of obese mothers and correlation with maternal insulin sensitivity," The Journal of Maternal-Fetal \& Neonatal Medicine, vol. 25, no. 11, pp. 2274-2280, 2012.

[24] Ü. Görkem, F. K. Küçükler, C. Toğrul, and T. Güngör, “Are adipokines associated with gestational diabetes mellitus?," Journal Of The Turkish-German Gynecological Association, vol. 17, no. 4, pp. 186-190, 2016.

[25] D. Pfau, H. Stepan, J. Kratzsch et al., "Circulating levels of the adipokine chemerin in gestational diabetes mellitus," Hormone Research in Peediatrics, vol. 74, no. 1, pp. 56-61, 2010.

[26] J. Shao, M. Liu, F. Li, and X. Xu, "The expression of chemerin in maternal peripheral, umbilical cord blood and placenta in gestational diabetic patients," Journal of Biomaterials and Tissue Engineering - American, vol. 5, no. 10, pp. 833-837, 2015.

[27] K. J. Hare, L. Bonde, J. A. Svare et al., "Decreased plasma chemerin levels in women with gestational diabetes mellitus," Diabetic Medicine, vol. 31, no. 8, pp. 936-940, 2014.

[28] L. H. Jørgensen, S. J. Petersson, J. Sellathurai et al., "Secreted protein acidic and rich in cysteine (SPARC) in human skeletal muscle," The Journal of Histochemistry and Cytochemistry, vol. 57, no. 1, pp. 29-39, 2009.

[29] S. L. Wong and M. B. Sukkar, "The SPARC protein: an overview of its role in lung cancer and pulmonary fibrosis and its potential role in chronic airways disease," British Journal of Pharmacology, vol. 174, no. 1, pp. 3-14, 2017.

[30] A. Chlenski and S. L. Cohn, "Modulation of matrix remodeling by SPARC in neoplastic progression," in Seminars in cell \& developmental biology, pp. 55-65, Elsevier, 2010.

[31] K. Kos, S. Wong, B. Tan et al., "Regulation of the fibrosis and angiogenesis promoter SPARC/osteonectin in human adipose tissue by weight change, leptin, insulin and glucose," Diabetes, vol. 58, no. 8, pp. 1780-1788, 2009.

[32] L. Xu, F. Ping, J. Yin et al., "Elevated plasma SPARC levels are associated with insulin resistance, dyslipidemia, and inflammation in gestational diabetes mellitus," PloS One, vol. 8, no. 12, article e81615, 2013.

[33] M. Kanauchi, H. Nishioka, and K. Dohi, "Secreted protein acidic and rich in cysteine (SPARC) in patients with diabetic nephropathy and tubulointerstitial injury," Diabetologia, vol. 43, no. 8, pp. 1076-1076, 2000.

[34] S. Kralisch and M. Fasshauer, "Adipocyte fatty acid binding protein: a novel adipokine involved in the pathogenesis of 
metabolic and vascular disease?," Diabetologia, vol. 56, no. 1, pp. 10-21, 2013.

[35] A. Xu, A. W. Tso, B. M. Cheung et al., "Circulating adipocytefatty acid binding protein levels predict the development of the metabolic syndrome," Circulation, vol. 115, no. 12, pp. 15371543, 2007.

[36] J. B. Boord, S. Fazio, and M. F. Linton, "Cytoplasmic fatty acidbinding proteins: emerging roles in metabolism and atherosclerosis," Current Opinion in Lipidology, vol. 13, no. 2, pp. 141-147, 2002.

[37] L. Makowski and G. S. Hotamisligil, "Fatty acid binding proteins - the evolutionary crossroads of inflammatory and metabolic responses," The Journal of Nutrition, vol. 134, no. 9, pp. 2464S-2468S, 2004.

[38] A. Xu, Y. Wang, J. Y. Xu et al., "Adipocyte fatty acid-binding protein is a plasma biomarker closely associated with obesity and metabolic syndrome," Clinical Chemistry, vol. 52, no. 3, pp. 405-413, 2006.

[39] C. H. Lee, C. Y. Y. Cheung, Y. C. Woo et al., "Circulating adipocyte fatty acid-binding protein concentrations predict multiple mortality outcomes among men and women with diabetes," Clinical Chemistry, vol. 64, no. 10, pp. 1496-1504, 2018.

[40] S. Kralisch, N. Klöting, T. Ebert et al., "Circulating adipocyte fatty acid-binding protein induces insulin resistance in mice in vivo," Obesity, vol. 23, no. 5, pp. 1007-1013, 2015.

[41] G. Niu, J. Li, H. Wang, Y. Ren, and J. Bai, "Associations of AFABP with anthropometric and metabolic indices and inflammatory cytokines in obese patients with newly diagnosed type 2 diabetes," BioMed Research International, vol. 2016, Article ID 9382092, 2016.

[42] H. Ortega-Senovilla, U. Schaefer-Graf, K. Meitzner et al., "Gestational diabetes mellitus causes changes in the concentrations of adipocyte fatty acid-binding protein and other adipocytokines in cord blood," Diabetes Care, vol. 34, no. 9, pp. 20612066, 2011.

[43] Y. Zhang, H. Zhang, and J. Lu, "Changes in serum adipocyte fatty acid-binding protein in women with gestational diabetes mellitus and normal pregnant women during mid- and late pregnancy," Journal of Diabetes Investigation, vol. 7, no. 5, pp. 797-804, 2016.

[44] M. Mosavat, S. Z. Omar, S. Jamalpour, and P. C. Tan, "Serum glucose-dependent insulinotropic polypeptide (GIP) and glucagon-like peptide-1 (GLP-1) in association with the risk of gestational diabetes: a prospective case-control study," Journal Diabetes Research, vol. 2020, pp. 1-7, 2020.

[45] D. Care, "Diagnosis and classification of diabetes mellitus," Diabetes Care, vol. 28, no. S37, pp. S5-10, 2006.

[46] S. Kralisch, H. Stepan, J. Kratzsch et al., "Serum levels of adipocyte fatty acid binding protein are increased in gestational diabetes mellitus," European Journal of Endocrinology, vol. 160, no. 1, pp. 33-38, 2009.

[47] M. Karpisek, D. Stejskal, H. Kotolova et al., "Treatment with atorvastatin reduces serum adipocyte-fatty acid binding protein value in patients with hyperlipidaemia," European Journal of Clinical Investigation, vol. 37, no. 8, pp. 637-642, 2007.

[48] D. Horakova, D. Stejskal, D. Pastucha, L. Muchova, and V. Janout, "Potential markers of insulin resistance in healthy vs obese and overweight subjects," Biomedical Papers of the Medical Faculty of the University Palacky, Olomouc, Czech Republic, vol. 154, no. 3, pp. 245-249, 2010.
[49] Y. Li, R. Xiao, C. Li, J. Huangfu, and J. Mao, "Increased plasma levels of FABP4 and PTEN are associated with more severe insulin resistance in women with gestational diabetes mellitus," Medical Science Monitor: International Medical Journal of Experimental and Clinical Research, vol. 21, p. 426, 2015.

[50] R. Bagheri, A. N. Qasim, N. N. Mehta et al., "Relation of plasma fatty acid binding proteins 4 and 5 with the metabolic syndrome, inflammation and coronary calcium in patients with type-2 diabetes mellitus," The American Journal of Cardiology, vol. 106, no. 8, pp. 1118-1123, 2010.

[51] H. Ortega-Senovilla, U. Schaefer-Graf, K. Meitzner et al., "Gestational diabetes mellitus causes changes in the concentrations of adipocyte fatty acid-binding protein and other adipocytokines in cord blood," Diabetes Care, vol. 34, no. 9, pp. 20612066, 2011.

[52] L. A. Barbour, C. E. McCurdy, T. L. Hernandez, J. P. Kirwan, P. M. Catalano, and J. E. Friedman, "Cellular mechanisms for insulin resistance in normal pregnancy and gestational diabetes," Diabetes Care, vol. 30, Supplement 2, pp. S112-S119, 2007.

[53] M. Takahashi, Y. Okimura, G. Iguchi et al., "Chemerin regulates $\beta$-cell function in mice," Scientific Reports, vol. 1, no. 1, p. 123, 2011.

[54] J. Brogin Moreli, A. M. Cirino Ruocco, J. M. Vernini, M. V. C. Rudge, and I. M. P. Calderon, "Interleukin 10 and tumor necrosis factor-alpha in pregnancy: aspects of interest in clinical obstetrics," ISRN Obstetrics and Gynecology, vol. 2012, 5 pages, 2012.

[55] M. F. Garces, E. Sanchez, B. J. Acosta et al., "Expression and regulation of chemerin during rat pregnancy," Placenta, vol. 33, no. 5, pp. 373-378, 2012.

[56] G. Valsamakis, S. Kumar, G. Creatsas, and G. Mastorakos, "The effects of adipose tissue and adipocytokines in human pregnancy," Annals of the New York Academy of Sciences, vol. 1205, no. 1, pp. 76-81, 2010. 